Ann. Biol. anim. Bioch. Biophys., I972, 12 (3), 479-49I.

\title{
HYPERMAGNÉSÉMIE ET SÉCRÉTION MÉDULLO-SURRÉNALIENNE (ํ)
}

\author{
Y. RAYSSIGUIER et P. LARVOR \\ Station de Physiopathologie de la Nutrition, \\ Centre de Recherches zootechniques et vétérinaires, I. N.R. A., \\ Theix, 63 - Saint-Genès-Champanelle
}

\begin{abstract}
RÉSUMÉ
L'injection d'adrénaline élève la magnésémie du Rat et on peut se demander si la médullosurrénale pourrait expliquer certaines hypermagnésémies observées lors de la stimulation de la sécrétion surrénalienne. La démédullation surrénalienne n'empêche pas l'hypermagnésémie due à l'anoxie, l'hémorragie, l'hypothermie, l'injection de morphine ou d'histamine; donc, dans ces conditions expérimentales, ce n'est pas la sécrétion d'adrénaline endogène qui modifie la magnésémie du Rat. Par contre, l'injection de pilocarpine ou de méthylcarbachol provoque une hypermagnésémie dépendante de la présence de la médullo-surrénale. Le traitement par l'atropine ou l'hexaméthonium n'inhibe pas l'hypermagnésémie après pilocarpine. Le mécanisme cholinergique de la sécrétion médullo-surrénalienne est connu mais le facteur hypermagnésémiant en cause ne paraît s'identifier ni à l'adrénaline, ni à la noradrénaline pour les raisons suivantes : l'activation de la sécrétion d'adrénaline par hypoglycémie insulinique n'élève pas la magnésémie. L'hypermagnésémie après pilocarpine peut être supprimée par le propranolol et renforcée par la phentolamine alors que les caractéristiques pharmacologiques du blocage de l'hypermagnésémie adrénalinique sont très différentes. Enfin le traitement par la réserpine n'empêche pas l'augmentation de la magnésémie après pilocarpine. L'hypothèse d'un facteur hypermagnésémiant médullosurrénalien est envisagée.
\end{abstract}

\section{INTRODUCTION}

L'injection de certaines substances adrénergiques peut entraîner une hypermagnésémie importante (LARVOR, I968; CLASSEN et al., I97I). L'adrénaline possède 1'action la plus puissante sur la magnésémie, la noradrénaline est inactive. En raison des fortes doses de catécholamines utilisées, le caractère physiologique d'un tel effet hypermagnésémiant reste incertain. Cependant, on connaît de nombreux facteurs qui induisent à la fois une forte libération d'adrénaline et une augmentation

(1) Une partie des résultats a été présentée au Ier Symposium international sur le déficit magnésique en pathologie humaine, Vittel, I97r. 
du magnésium plasmatique ; il est donc intéressant d'envisager la possibilité d'action des catécholamines endogènes. Lors d'hémorragie, d'hypoxie, d'hypercapnie ou d'hypothermie, par exemple, la sécrétion médullo-surrénalienne accrue pourrait être à l'origine des hypermagnésémies observées. Enfin diverses substances adrénalinosécrétrices comme la morphine et l'acétylcholine sont connues pour être hypermagnésémiantes.

Pour tester cette hypothèse, nous avons soumis des rats adréno-démédullés ou pseudo-opérés à divers agents adrénalino-sécréteurs : facteurs stimulant la sécrétion endogène (respiration dans une atmosphère confinée, hémorragie, hypothermie) ou substances pharmacologiques (morphine, insuline, histamine, pilocarpine et méthylcarbachol).

\section{MATÉRIEL, ET MÉTHODES}

Des rats de souche Sherman, non à jeun, des deux sexes et du même élevage sont répartis au hasard dans les groupes nécessaires aux essais. Pour une même expérience, ils sont choisis de même sexe et de poids voisin. Les rats sont adréno-démédullés selon la technique décrite par INGLE et GRIFFITH (1963) et sont utilisés, sauf indication contraire, un mois après l'opération. Pendant cette période de récupération, ils ne sont l'objet d'aucun soin particulier. Des animaux " pseudo-opérés " subissent un simulacre d'opération et sont maintenus dans les mêmes conditions avant leur utilisation. Le sang est prélevé par ponction cardiaque sous anesthésie à l'éther. Dans le plasma hépariné, on dose le magnésium par absorption atomique (Appareil PerkinElmer 303, Dawson et Heaton, r96r). Le glucose est déterminé par la glucose oxydase (Mrched, 1971).

\section{I. - Effet de divers facteurs stimulant la sécrétion endogène}

\section{Respiration en atmosphère confinée.}

Deux groupes de 6 rats mâles d'un poids moyen de 2 ro g sont enfermés dans deux enceintes hermétiquement closes d'une capacité de 6 litres. Pour éviter l'asphyxie, l'air est renouvelé rapidement 25 minutes après la fermeture du bocal. Les animaux sont retirés après une nouvelle période de 25 minutes de respiration en atmosphère confinée et ponctionnés aussitôt.

\section{Hémorragie.}

Une saignée représentant $3 p$. Ioo du poids du corps est réalisée rapidement par ponction cardiaque sous anesthésie à l'éther chez des rats mâles ou pseudo-opérés pesant environ $24^{\circ} \mathrm{g}$. Une nouvelle ponction cardiaque est réalisée 60 minutes après.

\section{Hypothermie.}

Des rats mâles d'un poids moyen de $25^{\circ} \mathrm{g}$ opérés ou pseudo-opérés sont placés dans des cages individuelles en matière plastique, maintenues à une température de - Io ${ }^{\circ} \mathrm{C}$ pendant $60 \mathrm{mi}-$ nutes et sont ponctionnés aussitôt après leur sortie de la chambre froide. Les rats témoins sont à la température de l'élevage $\left(22^{\circ} \mathrm{C}\right)$.

\section{2. - Effet de l'injection de substances pharmacologiques}

Le poids et le sexe des animaux sont précisés dans les différents essais. Comme substances adrénalino-sécrétrices, nous avons utilisé successivement : la morphine, l'histamine, l'insuline, le méthylcarbachol et la pilocarpine.

Les rats témoins reçoivent du sérum physiologique en quantité équivalente au volume injecté chez les animaux traités. Les substances sont administrées par voie intrapéritonéale à l'exception de la morphine par voie intraveineuse. Sauf indication contraire, les adrénolytiques (phentolamine et propranolol) et l'atropine sont injectés 30 minutes, l'hexaméthonium 15 minutes et la réserpine I6 heures, avant les injections de substances adrénalino-sécrétrices. Le sang est prélevé 6o minutes après ces dernières injections. 


\section{RÉSULTATS}

\section{I. - Effet de divers facteurs stimulant la sécrétion endogène}

La respiration en atmosphère confinée, 1'hémorragie ou l'exposition à basse température entraînent une élévation significative de la magnésémie aussi bien chez les rats adréno-démédullés que chez des rats pseudo-opérés 'comme le montre le tableau $\mathrm{r}$.

\section{TABLEAU I}

Effet de divers facteurs adrénalino-sécréteurs sur la magnésémie du Rat adréno-démédullé ou pseudo-opéré

Magnésémie $\left(\mathrm{mg} / 100 \mathrm{ml}\right.$ ). Moyenne \pm erreur type $\frac{\sigma}{\sqrt{\mathrm{N}}}$; entre parenthèses nombre d'animaux. La signification des différences est indiquée par un ou deux astérisques (respectivement $\mathrm{P}<0,05$ et $\mathrm{P}<0,0 \mathrm{I})$. La différence entre témoins et traités est indiquée dans le tableaului-même. La différence entre pseudo-opérés et opérés ou entre animaux avec ou sans injection préalable est indiquée dans la partie inférieure.

\section{Magnesaemia in the Rat after various factors and epinephrine release} effect of adrenal demedullation

Magnesaemia $(\mathrm{mg} / 100 \mathrm{ml})$. Mean $\pm \mathrm{S}$. E., between parentheses number of animals in each groups, one and two asteriks mean respectively $\mathrm{P}<0,05$ and $\mathrm{P}<0,0 \mathrm{I}$; the significance of the difference between controls and treated is shown in the table, for the difference between sham-operated and operated or between rats with or without previous injection the significance is shown in the lowest part of the table.

\begin{tabular}{|c|c|c|c|c|}
\hline & \multicolumn{2}{|c|}{ Témoins Controls } & \multicolumn{2}{|l|}{ Traités } \\
\hline & $\begin{array}{l}\text { Pseudo-opérés } \\
\text { Sham-operated }\end{array}$ & $\begin{array}{l}\text { Opérés } \\
\text { Operated }\end{array}$ & $\begin{array}{l}\text { Pseudo-opérés } \\
\text { Sham-operated }\end{array}$ & $\begin{array}{l}\text { Opérés } \\
\text { Operated }\end{array}$ \\
\hline $\begin{array}{l}\text { Hypoxie } \\
\text { Hypoxia }\end{array}$ & $1,50 \pm 0,04$ & $1,54 \underset{(6)}{ \pm} 0,02$ & $2,27 \underset{(6)}{ \pm 0,12^{* *}}$ & $2,39 \underset{(6)}{ \pm 0,17 * *}$ \\
\hline $\begin{array}{l}\text { Hémorragie } \\
\text { Hemorrage }\end{array}$ & $1,67 \underset{(14)}{ \pm} 0,04$ & $\underbrace{1,58 \pm 0,04}_{(12)}$ & $2,19 \pm \frac{ \pm}{(14)} 0,12 * *$ & $2,13 \pm 0,17^{* *}$ \\
\hline $\begin{array}{c}\text { Hypothermie } \\
\text { Hypothermia }\end{array}$ & $1,33 \frac{ \pm}{(7)} 0,02$ & $1,36 \frac{ \pm}{(7)} 0,03$ & $1,52 \underset{(8)}{ \pm} 0,04 * *$ & $1,53 \pm 0,05^{* *}$ \\
\hline $\begin{array}{l}\text { Signification } \\
\text { Significance }\end{array}$ & & & & \\
\hline
\end{tabular}

\section{2. - Effet de substances pharmacologiques}

\section{Morphine.}

L'injection de $8 \mathrm{mg} / \mathrm{kg}$ de chlorhydrate de morphine élève la magnésémie de la même façon chez des rats mâles opérés ou pseuđo-opérés pesant en moyenne I8o g. 


\section{Histamine.}

L'hypermagnésémie consécutive à l'injection de $150 \mathrm{mg} / \mathrm{kg}$ d'histamine chez des rats mâles de $200 \mathrm{~g}$ se retrouve chez des animaux sans médullo-surrénale. La magnésémie des rats témoins démédullés est légèrement plus faible dans cet essai que celle des animaux pseudo-opérés.

\section{TABLEAU 2}

Effet de l'injection de substances pharmacologiques adrénalino-sécrétrices sur la magnésémie du Rat advéno-démédullé ou pseudo-opéré

(Même expression des résultats que pour le tableau r)

Magnesaemia in the Rat after various injections and epinephrine release effect of adrenal demedullation

(Same expression of results than for table I)

\begin{tabular}{|c|c|c|c|c|}
\hline & Témoins & Controls & Traités & Treated \\
\hline & $\begin{array}{l}\text { Pseudo-opérés } \\
\text { Sham-operated }\end{array}$ & $\begin{array}{c}\text { Opérés } \\
\text { Operated }\end{array}$ & $\begin{array}{l}\text { Pseudo-opérés } \\
\text { Sham-operated }\end{array}$ & $\begin{array}{l}\text { Opérés } \\
\text { Operated }\end{array}$ \\
\hline Morphine & $1,52 \underset{(8)}{ \pm} 0,04$ & $1,49 \underset{(5)}{ \pm} 0,04$ & $2,03 \pm \underset{(8)}{0,06 * *}$ & $1,96 \underset{(5)}{ \pm} 0,06^{* *}$ \\
\hline Histamine & $1,32 \underset{(8)}{ \pm} 0,03$ & $1,20 \underset{(8)}{ \pm} 0,04$ & $1,72 \frac{1}{(8)} 0,07 * *$ & $1,64 \pm 0,14^{*}$ \\
\hline Insuline & $1,45 \underset{(8)}{ \pm} 0,03$ & $1,40 \underset{(8)}{ \pm} 0,03$ & $1,45 \underset{(8)}{ \pm} 0,05$ & $1,44 \underset{(4)}{ \pm} 0,04$ \\
\hline Méthylcarbachol & $1,41 \underset{(9)}{ \pm} 0,02$ & $1,49 \underset{(7)}{ \pm} 0,04$ & $2,07 \underset{(8)}{ \pm} 0,12 * *$ & $1,69 \underset{(7)}{ \pm} 0,07^{*}$ \\
\hline $\begin{array}{l}\text { Signification } \\
\text { Significance }\end{array}$ & & & \multicolumn{2}{|c|}{$\begin{array}{c}\text { NS } \\
\text { NS } \\
\text { NS } \\
*\end{array}$} \\
\hline
\end{tabular}

\section{Insuline.}

Chez des rats mâles d'un poids moyen de 2 Io g, la magnésémie n'est pas modifiée par l'injection de $40 \mathrm{UI} / \mathrm{kg}$ d'insuline.

\section{Méthylcarbachol.}

La magnésémie après 1'injection de $160 \mathrm{mg} / \mathrm{kg}$ de méthylcarbachol est significativement plus faible chez des rats Sherman femelles démédullés pesant $200 \mathrm{~g}$.

Le tableau 2 résume les résultats de ces différents essais.

\section{Pilocarpine.}

Chez des rats mâles pesant $280 \mathrm{~g}$ (tabl. 3) la pilocarpine à la dose de $200 \mathrm{mg} / \mathrm{kg}$ élève de façon hautement significative la magnésémie et la glycémie. Chez des rats privés de médullo-surrénale la magnésémie n'est pas augmentée tandis que l'hyper- 
TABLEAU 3

Effet d'une injection d'adrénaline (I $\mathrm{mg} / \mathrm{kg}$ ) ou de pilocarpine (200 $\mathrm{mg} / \mathrm{kg})$ sur la magnésémie et la glycémie du Rat et influence de l'ablation de la médullo-surrénale. (Glycémie $\mathrm{g} / \mathrm{l}$ )

(Même expression des résultats que pour le tableau $\mathrm{r}$ )

Blood plasma magnesium and glucose $60 \mathrm{mn}$ after adrenaline ( $\mathrm{r} \mathrm{mg} / \mathrm{kg})$ or pilocarpine $(200 \mathrm{mg} / \mathrm{kg})$ injection in Rats with or without adrenal demedullation

(Same expression of results than for table I)

\begin{tabular}{|c|c|c|c|c|c|c|}
\hline & Témoins & Controls & \multicolumn{2}{|c|}{ Adrénaline } & \multicolumn{2}{|c|}{ Pilocarpine } \\
\hline & Magnésium & Glucose & Magnésium & Glucose & Magnésium & Glucose \\
\hline $\begin{array}{l}\text { Pseudo-opérés } \\
\text { Sham-operated }\end{array}$ & $1,42 \underset{(8)}{ \pm} 0,03$ & $1,41 \underset{(8)}{ \pm} 0,04$ & $2,59 \underset{(8)}{ \pm} 0,21 * *$ & $3,95 \underset{(8)}{ \pm} 0,49 * *$ & $1,77 \underset{(8)}{ \pm} 0,06^{* *}$ & $3,26 \underset{(8)}{ \pm} 0,18^{* *}$ \\
\hline $\begin{array}{l}\text { Opérés } \\
\text { Operated }\end{array}$ & $1,50 \underset{(7)}{ \pm} 0,04$ & $1,26 \underset{(7)}{ \pm} 0,04$ & $3,00 \underset{(7)}{ \pm} 0,13^{* *}$ & $4,37 \underset{(7)}{ \pm} 0,12^{* *}$ & $1,46 \underset{(7)}{ \pm} 0,05^{\mathrm{Ns}}$ & $2,00 \underset{(7)}{ \pm} 0,17^{* *}$ \\
\hline $\begin{array}{l}\text { Signification } \\
\text { Significance }\end{array}$ & NS & * & NS & NS & $* *$ & $* *$ \\
\hline
\end{tabular}

\section{TABLEAU 4}

Effet de l'injection de divers bloqueurs pharmacologiques sur l'hypermagnésémie après pilocarpine

(Même expression des résultats que pour le tableau $I$ )

Magnesaemia in the Rat after pilocarpine with previous injection of various blocking agents (Same expression of results than table $\mathbf{r}$ )

\begin{tabular}{|c|c|c|c|c|}
\hline & Témoin & Controls & \multicolumn{2}{|c|}{ Pilocarpine } \\
\hline Bloqueurs & 0 & $\begin{array}{l}\text { Traités } \\
\text { Treated }\end{array}$ & 0 & $\begin{array}{l}\text { Traités } \\
\text { Treated }\end{array}$ \\
\hline Atropine & $1,73 \underset{(7)}{ \pm} 0,04$ & $1,79 \underset{(7)}{ \pm} 0,04$ & $2,05 \underset{(7)}{ \pm} 0,06^{* *}$ & $1,93 \underset{(7)}{ \pm} 0,04^{* *}$ \\
\hline Hexaméthonium & $1,66 \underset{(7)}{ \pm} 0,03$ & $1,67 \underset{(7)}{ \pm} 0,05$ & $1,88 \underset{\frac{1}{(7)}}{ \pm} 0,07^{* *}$ & $1,95 \underset{(7)}{ \pm} 0,07 * *$ \\
\hline Propranolol & $1, \mathbf{4 4} \underset{(7)}{ \pm} 0,03$ & $1,40 \underset{(7)}{ \pm} 0,04$ & $1,72 \underset{(8)}{ \pm} 0,05^{* *}$ & $1,41 \underset{(8)}{ \pm} 0,04^{\mathrm{NS}}$ \\
\hline Phentolamine & $1,42 \pm 0,03$ & $1,45 \underset{(7)}{ \pm} 0,03$ & $1,57 \frac{ \pm}{(7)} 0,04^{* *}$ & $2,19 \underset{(7)}{ \pm} 0,09^{* *}$ \\
\hline $\begin{array}{l}\text { Signification } \\
\text { Significance }\end{array}$ & \multicolumn{2}{|c|}{$\begin{array}{l}\text { NS } \\
\text { NS } \\
\text { NS } \\
\text { NS }\end{array}$} & \multicolumn{2}{|c|}{$\begin{array}{l}\text { NS } \\
\text { NS } \\
* * \\
* *\end{array}$} \\
\hline
\end{tabular}




\section{TABLEAU 5}

Magnésémie du Rat après injection d'adrénaline ( $\mathrm{I} \mathrm{mg} / \mathrm{kg}$ ) ou de pilocarpine (200 $\mathrm{mg} / \mathrm{kg}$ ) et influence de l'injection préalable de l'association phentolamine + propranolol (1o $+5 \mathrm{mg} / \mathrm{kg}$ )

(Même expression des résultats que pour le tableau I)

Magnesaemia in the Rat after adrenaline ( $\mathrm{I} \mathrm{mg} / \mathrm{kg}$ ) or pilocarpine (200 $\mathrm{mg} / \mathrm{kg})$ injection and infuence of a previous iniection

of a phentolamine + propranolol (1o $+5 \mathrm{mg} / \mathrm{kg}$ ) mixture

(Same expression of results than for table $\mathrm{I}$ )

\begin{tabular}{|c|c|c|c|}
\hline & Témoins Controls & Adrénaline & Pilocarpine \\
\hline 0 & $1,38 \underset{(6)}{ \pm} 0,04$ & $2,14 \pm \underset{(6)}{ \pm 0,10^{* *}}$ & $1,63 \pm 0,08 * *$ \\
\hline $\begin{array}{l}\text { Phentolamine } \\
+ \text { propranolol }\end{array}$ & $1,34 \underset{(6)}{ \pm} 0,02$ & $1,39 \underset{(6)}{ \pm 0,03^{\mathrm{NS}}}$ & $\frac{1,62 \pm 0,03^{* *}}{(6)}$ \\
\hline $\begin{array}{l}\text { Signification } \\
\text { Significance }\end{array}$ & NS & $* *$ & NS \\
\hline
\end{tabular}

glycémie est significativement plus faible. L'injection d'adrénaline provoque une hypermagnésémie et une hyperglycémie à la fois chez les opérés et pseudo-opérés.

Des résultats semblables à ceux du tableau 3 ont été obtenus chez des rats utilisés aussitôt après une démédullation ou une pseudo-opération.

Les injections d'atropine (Ioo $\mathrm{mg} / \mathrm{kg}$ ) ou d'hexaméthonium (25 $\mathrm{mg} / \mathrm{kg}$ ) sont sans effet sur 1'hypermagnésémie due à la pilocarpine chez des rats femelles de I6o $\mathrm{g}$. Le propranolol à la dose de $5 \mathrm{mg} / \mathrm{kg}$ permet d'empêcher l'hypermagnésémie pour

\section{TABLEAU 6}

Magnésémie du Rat après injection de pilocarpine (200 $\mathrm{mg} / \mathrm{kg})$ et de phentolamine (Io $\mathrm{mg} / \mathrm{kg}$ ) et infuence de l'ablation de la médullo-surrénale

(Même expression des résultats que pour le tableau I)

Magnesaemia in the Rat after pilocarpine $(200 \mathrm{mg} / \mathrm{kg}$ ) and phentolamine (10 $\mathrm{mg} / \mathrm{kg}$ ) injection and effect of adrenal demedullation (Same expression of results than for table $\mathbf{I}$ )

\begin{tabular}{c|c|c}
\hline \hline & Témoins Controls & Témoins Treated \\
\hline $\begin{array}{c}\text { Pseudo-opérés } \\
\text { Sham-operated }\end{array}$ & $\begin{array}{c}1,55 \pm 0,05 \\
(6)\end{array}$ & $\begin{array}{c}2,15 \pm 0,11^{* *} \\
(9)\end{array}$ \\
\hline $\begin{array}{c}\text { Opérés } \\
\text { Operated }\end{array}$ & $\begin{array}{c} \pm 0,05 \mathrm{Ns} \\
(6)\end{array}$ \\
\hline $\begin{array}{c}\text { Signification } \\
\text { Significance }\end{array}$ & $\mathrm{NS}$ & $*, 04$ \\
\hline \hline
\end{tabular}


des rats mâles de $I_{50} \mathrm{~g}$ environ, tandis que la phentolamine (Io $\mathrm{mg} / \mathrm{kg}$ ) renforce l'effet hypermagnésémiant de la pilocarpine chez des rats mâles de I30 g (tab1. 4). L'effet de l'association de ces deux derniers bloqueurs adrénergiques est indiqué dans le tableau 5, pour des rats femelles de $180 \mathrm{~g}$ vis-à-vis de 1 'hypermagnésémie après adrénaline ou pilocarpine. L'hypermagnésémie due à l'injection simultanée de phentolamine et de pilocarpine est inhibée par la démédullation des animaux (rats femelles de $220 \mathrm{~g}$, tabl. 6) mais non par l'injection préalable de $5 \mathrm{mg} / \mathrm{kg}$ de réserpine (rats mâles de $120 \mathrm{~g}$, tabl. 7).

\section{TABLEAU 7}

Magnésémie du Rat 60 mn après l'injection de l'association pilocarpine et phentolamine $(20+5 \mathrm{mg} / \mathrm{kg})$ et infuence de l'injection préalable de réserpine $(5 \mathrm{mg} / \mathrm{kg}), 16$ heures auparavant

(Même expression des résultats que pour le tableau I)

Magnesaemia in the Rat $60 \mathrm{mn}$ after pilocarpine

and phentolamine injection $(20+5 \mathrm{mg} / \mathrm{kg})$

with or without injection of reserpine (Io $\mathrm{mg} / \mathrm{kg}$ ) $16 \mathrm{~h}$ before

(Same expression of results than for table $I$ )

\begin{tabular}{c|c|c|c}
\hline \hline & Témoins Controls & Phentolamine & $\begin{array}{c}\text { Pilocarpine } \\
\text { phentolamine }\end{array}$ \\
\hline 0 & $\begin{array}{c}1,54 \pm 0,05 \\
(7)\end{array}$ & $\begin{array}{c}1,49 \pm 0,05 \\
(7)\end{array}$ & $\begin{array}{c}2,02 \pm 0,07^{* *} \\
(14)\end{array}$ \\
\hline Réserpine & $1,41 \pm 0,05$ & $\begin{array}{r}1,45 \frac{ \pm 0,04}{(6)} \\
(6)\end{array}$ & $\begin{array}{c}1,95 \pm 0,07^{* *} \\
(13)\end{array}$ \\
\hline $\begin{array}{l}\text { Signification } \\
\text { Significance }\end{array}$ & NS & NS & NS \\
\hline \hline
\end{tabular}

\section{DISCUSSION}

Généralement la surrénalectomie totale est suivie de perturbations de la magnésémie difficiles à interpréter du fait du caractère composite des glandes surrénales (voir discussion in LARVOR et DURLACH, I97I).

Dans nos essais, les rats étaient utilisés un mois après l'ablation de la médulla et pendant cette période ils n'étaient l'objet d'aucun soin particulier. De l'eau non salée était à leur disposition. Le cortex surrénalien restait donc suffisamment fonctionnel (GAUNT et al., I968), même si une partie de la zone corticale était arrachée avec la médulla au moment de l'énucléation; l'autopsie montrait d'ailleurs une large régénération $\mathrm{d} u$ cortex chez ces rats.

La magnésie des animaux sans médullo-surrénale n'était la plupart du temps pas différente de celle des animaux pseudo-opérés (le tableau 8 permet de comparer l'ensemble des résultats). Ces résultats diffèrent de ceux obtenus avec une alimentation carencée en magnésium (LARVOR et RAySSIGUIER, I97I $a$ ) puisque dans ce dernier cas 1'ablation des médullo-surrénales entraîne une aggravation de la carence. 
Asphyxie.

S'il est bien établi depuis Cannon et Hoskrns (IgII) qu'une hypoxie sévère entraîne un accroissement de la sécrétion médullo-surrénalienne, les travaux concernant la magnésémie et l'hypoxie sont plus fragmentaires.

\section{TABLEAU 8}

Magnésémie du Rat pseudo-opéré ou advéno-démédullé.

Regroupement des données provenant de différentes expériences (tabl. I, 2, 3,6)

(Même expression des résultats que pour le tableau I)

Magnesaemia in the Rat following sham operation or adrenal demedullation.

Collection of data extracted from tables I, 2, 3, 6

(Same expression of results than for table $\mathrm{I}$ )

\begin{tabular}{|c|c|c|c|c|c|c|c|c|c|c|}
\hline No du tableau & 1 & 1 & 1 & 2 & 2 & 2 & 2 & 3 & 6 & Ensemble \\
\hline $\begin{array}{l}\text { Pseudo-opérés } \\
\text { Sham-operated }\end{array}$ & $\begin{array}{c}1,50 \\
\pm \\
0,04 \\
(6)\end{array}$ & $\begin{array}{c}1,67 \\
\pm \\
0,0^{\prime} \\
(14)\end{array}$ & $\begin{array}{c}1,33 \\
\pm \\
0,02 \\
(7)\end{array}$ & $\begin{array}{c}1,52 \\
\pm \\
0,04 \\
(8)\end{array}$ & $\begin{array}{c}1,32 \\
\pm \\
0,03 \\
(8)\end{array}$ & $\begin{array}{c}1,45 \\
\pm \\
0,03 \\
(8)\end{array}$ & $\begin{array}{c}1,41 \\
\pm \\
0,02 \\
(9)\end{array}$ & $\begin{array}{c}1,42 \\
\pm \\
0,03 \\
(8)\end{array}$ & $\begin{array}{c}1,55 \\
\pm \\
0,04 \\
(6)\end{array}$ & 1,46 \\
\hline $\begin{array}{c}\text { Opérés } \\
\text { operated }\end{array}$ & $\begin{array}{c}1,54 \\
\pm \\
0,02 \\
(6)\end{array}$ & $\begin{array}{c}1,58 \\
\pm \\
0,04 \\
(12)\end{array}$ & $\begin{array}{c}1,36 \\
\pm \\
\mathbf{0 , 0 3} \\
(7)\end{array}$ & $\begin{array}{c}1,49 \\
\pm \\
0,04 \\
(5)\end{array}$ & $\begin{array}{c}1,20 \\
\pm \\
0,04 \\
(8)\end{array}$ & $\begin{array}{c}1,40 \\
\pm \\
0,03 \\
(8)\end{array}$ & $\begin{array}{c}1,49 \\
\pm \\
0,04 \\
(7)\end{array}$ & $\begin{array}{c}1,50 \\
\pm \\
0,04 \\
(7)\end{array}$ & $\begin{array}{c}1,52 \\
\pm \\
\mathbf{0 , 0 5} \\
(5)\end{array}$ & 1,45 \\
\hline $\begin{array}{l}\text { Signification } \\
\text { Significance }\end{array}$ & NS & NS & NS & NS & * & NS & NS & NS & NS & NS \\
\hline
\end{tabular}

L'acidose respiratoire s'accompagne d'une augmentation du taux du magnésium plasmatique chez le Chien (Maurat et al., I964). Chez le Rat l'exposition à une atmosphère hypercapnique élève la magnésémie (BANGE et al., I964). Une corrélation est observée entre les signes d'asphyxie à la naissance et la magnésémie chez le nouveau-né (ENGEL, et EILIN, I970).

Dans notre essai, les animaux se trouvent dans une atmosphère hypoxique et hypercapnique et nous obtenons une élévation de plus de $50 \mathrm{p}$. roo du magnésium plasmatique à la fois chez les opérés et les pseudo-opérés.

\section{Hémorragie.}

En cas d'hémorragie une hypermagnésémie a été décrite (GoLDSMITH, I969) tandis que 1'hypersécrétion de la médulla est une donnée classique, mais les animaux démédullés et pseudo-opérés manifestent la même hypermagnésémie; 1à non plus on ne peut conclure à une relation de causalité entre ces deux phénomènes.

\section{Exposition au froid.}

NeUBEISER et al., Ig6I signalent une élévation de la magnésémie chez le Rat après une première journée d'exposition à $+4^{\circ} \mathrm{C}$. Dans nos conditions expérimen- 
tales, la modification de la magnésémie est un phénomène rapide en rapport avec une exposition au froid sévère $\left(-\mathrm{IO}^{\circ} \mathrm{C}\right)$. La production, la libération et l'élimination urinaires de catécholamines sont accrues lors de l'adaptation du Rat à basse température (LEDUC, I96I ; BERTIN et CHEVILLARD, I967) mais la noradrénaline semble jouer le rôle principal lors de l'adaptation au froid (LEBLANC et PouLIOT, I964).

Dans notre essai, l'ablation de la médullo-surrénale n'entraîne pas une diminution de la résistance et de la survie au froid (à court terme). L'augmentation de la magnésémie est identique dans les deux groupes d'animaux.

\section{Effets de substances pharmacologiques}

Morphine.

Parmi les substances pharmacologiques hypermagnésémiantes, la morphine (VACHON et MARCHAND, I970) est aussi connue pour stimuler la sécrétion de la médullosurrénale (OUTSCHOORN, I952) mais l'hypermagnésémie obtenue n'est pas supprimée par la démédullation.

\section{Histamine.}

Les relations entre histamine et métabolisme du magnésium ont été envisagées en particulier lors de recherches sur l'étiologie de la Tétanie d'herbage. Selon GEELEN et al. (Ig66), la perfusion d'histamine ne modifie pas la magnésémie de la Vache laitière. Nous montrons chez le Rat qu'une forte injection d'histamine est hypermagnésémiante. L'adrénalino-sécrétion (DE SCHAEPDRYVER, I963) n'explique pas la réaction obtenue puisque les démédullés fournissent la même réponse.

\section{Insuline.}

Le choc hypoglycémique est l'un des stimulants les plus puissants de la sécrétion médullo-surrénalienne (VON EULER et LUFT, I952) ce qui correspond aux conditions recherchées dans cette étude. L'effet de l'insuline sur le taux du magnésium plasmatique a donné lieu à des travaux cliniques et expérimentaux dont les résultats ne sont pas toujours concordants. Pour VALENCIA (I955) au cours de l'hypoglycémie insulinique il y a une élévation de la magnésémie chez le Lapin. BERTHAUX et MAURAT (I958) obtiennent une hypomagnésémie chez le Chien sous l'effet combiné de l'insuline et du glucose mais dans ce cas la glycémie est maintenue au voisinage des taux physiologiques.

Au cours de nos essais l'insuline (sans glucose) n'a eu aucun effet sur la magnésémie du Rat, que ce soit en présence ou en 1'absence de la médullo-surrénale.

\section{Méthylcarbachol et pilocarpine.}

Le mécanisme cholinergique de la sécrétion médullo-surrénalienne nous a conduit à étudier l'action de ces substances d'autant plus que TERKILDSEN (I952) a montré l'effet hypermagnésémiant de l'acétylcholine ; au cours d'essais préliminaires non publiés, nous avons retrouvé cette action chez le Rat, mais sous une forme très fugace, par suite de l'action rapide des cholinestérases. Nous avons donc repris ces 
essais avec le méthylcarbachol et la pilocarpine, deux vagomimétiques beaucoup moins sensibles à la destruction enzymatique. Avec ces deux substances on observe régulièrement une hypermagnésémie significative dépendante de la présence de la médullo-surrénale. La surrénale paraît bien jouer le rôle de relais sécrétoire et non avoir une action simplement permissive, puisque la surrénalectomie réalisée juste avant l'injection de pilocarpine empêche l'hypermagnésémie.

L'action des agents vagomimétiques peut être classée (DAIE, I9I4) en action muscarinique (action vagomimétique proprement dite) que l'on peut bloquer au moyen de 1'atropine, et en action nicotinique (stimulation des relais ganglionnaires sympathiques à fonctionnement cholinergique) que l'on peut bloquer au moyen de ganglioplégiques comme l'hexaméthonium, ou en vidant les réserves de catécholamines de l'organisme par la réserpine.

Il ne nous a pas été possible de faire entrer de façon certaine le mode d'action de la pilocarpine sur la magnésémie dans l'une de ces catégories. Chez les rats traités à la pilocarpine, l'atropine empêche toute apparition de symptômes muscariniques (augmentation des sécrétions glandulaires, diarrhée) mais ne modifie pas la magnésémie ce qui semble en faveur d'un effet hypermagnésémiant de type nicotinique. L'absence d'action de l'hexaméthonium et de la réserpine reste plus difficile à interpréter. La pilocarpine est capable de stimuler la médullo-surrénale et préférentiellement la sécrétion d'adrénaline (DOUGLas et PoISNER, I965) mais bien que 1'hypermagnésémie soit inhibée totalement par la démédullation, la sécrétion surrénalienne de noradrénaline et d'ađrénaline ne peut expliquer l'effet obtenu après pilocarpine. En effet, la noradrénaline est sans action sur la magnésémie (LARVor, I968) et l'action de l'adrénaline ne peut être bloquée efficacement que par l'association de phentolamine (bloqueur des $\alpha$-récepteurs adrénergiques) et de propranolol (bloqueur des $\beta$ - récepteurs adrénergiques) (LARVOR et RAYSSIGUIER, I97I $b$ ) contrairement à l'effet de la pilocarpine inhibé par le propranolol et potentialisé par la phentolamine.

L'absence d'hypermagnésémie après l'action de moyens hypoglycémiants très puissants (insuline) confirme qu'il ne peut être question d'une hypermagnésémie ayant l'adrénaline endogène pour médiateur.

La cortico-surrénale régénérée d'un animal démédullé ne peut être considérée comme tout à fait normale, mais la possibilité d'une modification de la sécrétion du cortex ne semble pas devoir être retenue : nos résultats sont identiques chez des rats injectés un mois après la démédullation ou utilisés dès la fin de l'énucléation.

\section{CONCLUSION}

Après mise en jeu de stress sévères (hypoxie, hémorragie, exposition à basse température), la magnésémie du Rat s'élève par un mécanisme qui ne fait pas intervenir la médullo-surrénale. Rien ne permet d'ailleurs de penser que ce mécanisme est le même dans tous les cas.

Il est par ailleurs, évident que, dans nos conditions d'expérience, la sécrétion endogène de catécholamines par la médullo-surrénale ne peut modifier la magnésémie, et à plus forte raison que cette sécrétion ne peut assurer l'homéostasie de $\mathrm{Mg}$.

Néanmoins, la médullo-surrénale est indispensable à la manifestation de l'hyper- 
magnésémie provoquée par des substances cholinergiques telles que la pilocarpine ou le méthylcarbachol.

Deux hypothèses logiques paraissent possibles quant à la nature de cette action, et nous ne voyons pas la possibilité d'une troisième solution :

- Ou la médullo-surrénale agit par sa simple présence, en créant dans l'organisme un état réceptif qui disparaît après la démédullation. Cette hypothèse d'une action permissive nous paraît exclue du fait que la surrénalectomie inhibe instantanément la réaction d'hypermagnésémie à la pilocarpine.

- Ou la médullo-surrénale est un maillon nécessaire dans la chaîne de réactions qui va de l'injection de pilocarpine à l'hypermagnésémie.

La vraisemblance de ce deuxième terme de l'alternative nous conduit à postuler l'existence dans la médullo-surrénale d'un facteur hypermagnésémiant, mis en jeu sous l'influence de la pilocarpine et différent del'adrénaline ou de la noradrénaline.

Reçu pour publication en février 1972.

\section{SUMMARY}

\section{HYPERMAGNESEMIA AND MEDUI,LO-ADRENAL, SECRETION}

Contrary to noradrenalin which does not produce magnesemia, the intraperitoneal injection of adrenalin causes severe hypermagnesemia in the rat. Some factors induce both endogenous catecholamine liberation and hypermagnesemia, and it is possible that increased medullo-adrenal secretion may explain the magnesemia modifications obtained in these conditions.

We used various adrenalino-secreting agents on rats with the medullo-adrenal excised or on those having a simulated operation. Blood was sampled by cardiac puncture under ether anethesia. In the heparinized plasma magnesium was determined by atomic absorption, and glucose by glucose oxydase.

\section{Effect of various factors stimulating endogenous secretion}

Hypermagnesemia are observed after 50 minutes of respiration in a confined atmosphere, 60 minutes after a severe hemorrage representing $3 \mathrm{p}$. I 00 of body weight, and after a one-hour exposure at a low temperature $\left(-\mathrm{I}^{\circ} \mathrm{C}\right)$ (table $\left.\mathrm{I}\right)$. In all cases, normally accompanied by increased medullo-adrenalin secretion, there is an elevation of magnesemia in the medullo-adrenal excised rats as well as in those having undergone the simulated operation.

\section{Effect of injecting pharmacological substances}

Unless otherwise indicated, substances are administered by intraperitoneal pathway. Adrenolytics and atropin are injected 30 minutes before the adrenalino-secreting substances are injected, and hexamethonium and reserpin I 5 minutes and $\mathrm{I} 6$ hours, respectively, before. Blood samples are taken $\mathrm{I}$ hour after the adrenalino-secreting injections.

Intravenous injections of morphine and histamine, known to be adrenalino-secreting agents, are hypermagnesemiant both in operated animals and in those having undergone simulated operations (Table 2). The hypoglycemic insulin shock, one of the most powerful stimulants of adrenalino secretion, had no effect on rat magnesemia (Table 2). The cholinergic mechanism of medullo-adrenal secretion led us to study methylcarbachol and pilocarpin action. Hypermagnesemia occurs with these two substances except in animals with the medulla excised (tables 2 and 3). Results similar to those in table 3 were obtained in rats used immediately after medulla excision or simulated operation. Atropin or hexamethonium injections have no effect on hypermagnesemia due to pilocarpin. Propranolol inhibits while phentolamine reinforces this hyper- 
magnesemic effect (table 4). The result of associating these two latter adrenergic blocking agents is shown in table 5 .

Hypermagnesemia due to the simultaneous injection of phentolamine and pilocarpin is inhibited by excising the medulla of the animals (table 6), but not by previous reserpin injection (table 7).

There are several main point in these results to note. The control rats were used one month after medulla excision and most of the time their magnesemia was not different from that of the animals having undergone simulated operations. These results differ from those obtained with a magnesium-deficient diet since excision of the medulla in the latter case aggravates the deficiency. (LARVor and RAYSSIGUIER I97I $a$ )

After severe stress (hypoxia, hemorrage, exposure to low temperature), a rise in rat magnesemia is caused by a mechanism which is not a result of medullo adrenal action, and in the experimental conditions studied, endogenous adrenalin secretion does not control magnesemia.

Hypermagnesemia dependent on the presence of the medullo-adrenal can only be obtained by using adrenalin-secreting agents such as vagomimetic substances like methylcarbachol or pilocarpin. The action of these substances is usually classed as muscarinic action (properly called vagomimetic action) which can be blocked with atropin, and nicotinic action (stimulation of sympathetic ganglion relays having cholinergic function) which can be blocked by ganglioplegics such as hexamethonium or by emptying stored catecholamines with reserpin. The effect of pilocarpin on magnesemia does not seem to belong to one of these categories. (It is not blocked by atropin, hexamethonium or reserpin.) Hypermagnesemiant pilocarpin action may be inhibited by medulla excision or by blocking adrenergic $\beta$ - receivers, and it may be potentialized by blocking adrenergic $\alpha$ - receivers. However, the hypermagnesemic factor in question cannot be identified with adrenalin or noradrenalin because noradrenalin does not act on magnesemia and the hypermagnesemiant action of adrenalin cannot be blocked by propranolol alone.

The absence of hypermagnesemia after hypoglycemia, and the fact that reserpin does not act in hypermagnesemia after pilocarpin seem to show that hypermagnesemia cannot have endogenous adrenalin as a mediator.

\section{RÉFÉRENCES BIBLIOGRAPHIQUES}

Bange C., Faye J.-L., Pêrès G., rg66. Influence de l'hypercapnie sur le taux du magnésium plasmatique et ses relations avec la glycémie. Étude chez le rat. $J$. Physiol. (Paris), 58, rog-r r4.

Berthaux P., Maurat J.-P., I958. Variations du magnésium plasmatique sous l'effet combiné de l'insuline et du glucose chez le chien. C. R. Soc. Biol., 152, 487-489.

Bertin R., Chevillard L., I967. Élimination urinaire des catécholamines chez le rat Long Evans au cours de l'adaptation à diverses températures. C. R. Soc. Biol., 161, 248-25I.

Cannon W. B., Hoskins R. G., IgII. The effect of asphyxia hyperpnoea and sensory stimulation on adrenal secretion. Am. J. Phys., 24, 274-279.

Classen H. G., Marquardt P., Spät H. M., Schumacher K. A., I971. Hypermagnesemia following exposure to acute stress. Pharmacology, 5, 287-294.

DALE H. H., r9r4. The action of certain esters and ethers of choline and their relation to muscarine. J. Pharmacol. Exptl. Therap., 6, 147-190.

Dawson J. B., Heaton E. W., r960. The determination of magnesium in biological materials by atomic absorption spectrophotometry. Biochem. $J ., 80$, 99-106.

De Schaepdryver A. F., I963. Pharmacologie de la transmission sympathique périphérique. Actualités pharmacol, 15, 224-255.

Douglas W. W., Porsner A. M., r965. Preferential release of adrenaline from the adrenal medulla by muscarine and pilocarpine. Nature, 208, I IO2-I IO3.

ENGEL R. R., Elin R. J., r97o. Hypermagnesemia from birth asphyxia. Pediatrics, 7r7, $63 \mathrm{I}-637$.

Gaunt R., Gisoldi E., Herkner J., Howie N., Renzi A. A., I968. Sodium retention after adrenal enucleation : drug and salt appetite studies. Endocrinology, 83, 927-932.

Geelen M. J. H., Van Rheenen D. L., Hendriks H. J., Seekles L., I966. Histamine in the blood of dairy cows in connection with nutrition tetany (grass tetany). Tijdschr. Diergeneesk., 91, 1577-I588.

Goldsmith N. F., HUGged H., URY H. K., Serum electrolytes and clotting factors in rats during blood loss by cardiac puncture: effect of norethynodrel with mestranol on serum magnesium. Rev. suisse Zool., 76, 849-864.

INGLE D. J., GRIFFTTH J. Q., I963. Surgery of the rat in : The rat in labovatory investigation, p. 43445I, I vol, $2^{\mathrm{e}}$ édition, Farris E. J., Griffith J. Q., editors. Publisher Hafner Publ. Co. New York. 
LARvor P., I968. Effet de l'adrénaline et de la noradrénaline sur la magnésémie et la glycémie du rat. Interférence de la dihydroergotamine et de l'insuline. Ann. Biol. anim. Bioch. Biophys., 8, $46 \mathrm{I}-464$.

Larvor P., Durlach J., 197r. Rapports physiologiques entre magnésium et glandes endocrines, p. 251-296. I Ier Symposium international sur le déficit magnésique en pathologie humaine, Vittel.

LARVor P. et RAyssiguier Y., Ig7r $a$. Influence de la démédullation surrénalienne sur le métabolisme du magnésium au cours de la carence en cet élément chez le rat. C. R. Acad. Sci., Paris, 273, 408-4I0.

LaRvor P., Rayssiguier Y., I97 $b$. Les récepteurs responsables de l'hypermagnésémie consécutive à une injection de catécholamines. Ann. Biol. anim. Bioch. Biophys., 11, 55-68,

Leblanc J., Pouliot M., I964. Importance of noradrenaline in cold adaptation. Am. J. Physiol., 207, $853-856$.

Leduc J., I96I. Catecholamine production and release in exposure and acclimatation to cold. Acta Physiol. Scand., 53, supp. 183 , I.

Maurat J.-P., Pocidalo J.-J., Lissac J., I964. Le magnésium plasmatique au cours des modifications de l'équilibre acido-basique. C. R. Soc. Biol., 158, 2 303-2 305.

Michel M.-C., I97I. Analyse quantitative de quelques substances azotes et glucidiques en milieu biologique. Essai de rationalisation p. 59-66. These, Fac. Sci. Clermont-Ferrand.

Neubeiser R. E., Platner W. Q., Shields J. L., I96r. Magnesium in blood and tissues during cold acclimatation. J. Appl. Physiol., 16, 247-249.

Outschoorn A. S., I952. The hormones of the adrenal medulla and their release. Brit. J. Pharmacol., 7, 605-6I5.

TERKILDSEN T. C., I952. The effect of physostigmine, neostigmine and acetylcholine on serum magnesium concentration. Acta Pharmacol., 8, 385-390.

Vachon M., MaRchand C., I970. The influence of morphine on magnesium metabolism in rats. J. Pharmacol. Exp. Ther., 172, 122-2I7.

VALENCra R., I955. Sur les variations des teneurs en sodium, potassium et magnésium plasmatiques sous l'effet de l'insuline. C. R. Acad. Sci., 241, 244-246.

VoN EULER U. S., LUFT R., I952. Effect of insulin on urinary excretion of adrenalin and noradrenalin. Metabolism, 1, 528-532. 